\title{
Is the ditio-octonoate monomer the ideal for adhesion to precious metals and their alloys?
}

\begin{abstract}
The use of metals in dentistry presupposes the need for mechanisms of retention or adhesion of the same to different substrates; Such as enamel, dentin, ceramics, old resins and other metals. ${ }^{1}$ Throughout history these mechanisms have been changing and evolving, from the purely mechanical retention to the present mechanisms of adhesion. ${ }^{2,3}$ It is in the decade of the 70 when they begin to develop adhesion materials. Prior to this, retention was basically mechanical, having also evolved from macromechanical retention systems to micromechanical retention systems. ${ }^{3}$ It is at the end of the 70 when the first generation of adhesives appears; These monomers were 4-META (4-methacryloyloxyethyl trimellitate anhydride) and 10-MDP (10-methacryloyloxydecyl dihydrogen phosphate). These were effective for use with non-precious metal alloys. ${ }^{4}$ At the end of the 80 appears the second generation of adhesives, which were effective with precious or noble metal alloys. The best known was VBATDT (4-vinylbenzyl-n-propyl). The new development of phosphonic acid monomers improves attachment to enamel and dentin. ${ }^{5}$ These could be used on a wide variety of precious and non-precious metal alloys. The optimum combination for bonding to hard and precious and non-precious precious metals and alloys was $5.0 \%$ by weight of $10 \mathrm{MDT}$ and $1.0 \%$ by weight of 6-MHPA phosphonoacetate. ${ }^{6}$ For bonding to dental porcelain, alumina, zirconia and gold alloy, a ternary combination of silane, acidic adhesive monomers and dithio-octonoate monomers appears promising. The most recent development was a multi-purpose self-etching adhesive containing only acid monomers and dihiooctonoate adhesive monomers, producing strong adhesion to enamel and dentin, zirconium oxide and gold alloys. ${ }^{1}$
\end{abstract}

Keywords: operative dentistry, minimally invasive dentistry, laser fluorescence, nanotechnology, laser ER, LLLT, adhesive monomer, dental alloys, metal primer, multi - purpose adhesive, $4-$ meta, $10-\mathrm{mdp}$
Volume 3 Issue I - 2017

\author{
Marcos Moradas Estrada \\ Department of Surgery and Surgical Medical Specialties, \\ University of Oviedo, Spain
}

\begin{abstract}
Correspondence: Marcos Moradas Estrada, Associate professor, Service of Conservative Dentistry and Dental Materials. Department of Surgery and Surgical Medical Specialties, University of Oviedo, Spain, Email marcosmords@gmail.com
\end{abstract}

Received: May 17, 2017 | Published: June 01, 2017

\section{Material and methods}

A descriptive bibliographic review of the evidence provided in indexed articles and other bibliographic sources, such as books, theses and others, has been made. These were analyzed and after checking whether or not they met the inclusion/exclusion criteria of this work, finally were 27 articles of bibliographic review published in a hairpin that goes from 2007 to 2016 .

\section{Introduction}

The use of metals in dentistry has great therapeutic advantages in many fields of dentistry, such as orthodontics, prostheses, conservative dentistry since its origins and more recently Implantology. ${ }^{2}$ Likewise, metals can be used on numerous and varied substrates: enamel, dentin, ceramics, resins or other metals. ${ }^{1}$ Examples of adhesion of enamel or dentin to metals, orthodontic bands and brackets, or Maryland type adhesive bridges. It may be necessary to attach a metal to ceramics in the case of a metal crown, which by aesthetics will be covered, or another metal (such as a cast post) to the metal of the crown and/or ceramics thereof, or even when it is free of metal. ${ }^{4}$

The first and foremost objective of dental adhesives is the longlasting attachment to their substrates or adhesives, whether hard dental tissues or a wide range of materials (some new and others already contaminated when already in the mouth). The adhesive system, already discovered by Bouncoure and Bowen in the 1960s, has meant an improvement in speed, efficiency and convenience by simplifying the joining steps of two bodies of disparate origin and nature and reducing the time in the clinician's working time. The current trend in the development of dental adhesive systems follows the line of selfetch adhesives, multipurpose primers or adhesives that give a strong and durable adhesion indiscriminately to a multitude of adherents that coexist in the oral environment, ranging from tejdios (Enamel and dentin), precious metals (noble), alloys of precious metals and alloys of non-precious metals to dental porcelain, ceramics based on aluminum oxide (alumina) and zirconium-based ceramics. ${ }^{6,7}$

Do not overlook the many detractors of self-etch adhesives. On the one hand self-etching adhesives are easy to use and less prone to cause postoperative sensitivity. Although on the other hand, they produce less enamel bond strength compared to phosphoric acid. ${ }^{5,6}$ In order to improve bonding to enamel as well as to non-noble metals, the adhesion promoter monomers (adhesive monomers) which have been developed and continue to be developed are most effective. To improve adhesion to precious metals, the sulfur-containing monomers were developed and the research efforts do not cease in the search for monomers containing modified sulfur.

As with all dental restorations, the long-term clinical success of ceramic restorations is based on a strong and durable resin bonding of the ceramic restoration completed to the supporting structure of the teeth. ${ }^{5,8}$ However, the establishment of a strong and stable bond with zirconium oxide using conventional surface roughness technologies and coating methods has proven difficult. 
Throughout the development of dental adhesives, several categories of monomers have been developed using technologies used in synthetic organic chemistry. One category are the monomers bearing the carboxyl group and its anhydride group ${ }^{5}$ or the phosphonic acid group $^{8}$ for bonding to hard dental tissues, non-precious metal alloys and dental porcelains; Another category are the sulfur-containing monomers bearing the Dithio-octonoate group ${ }^{8,9}$ for bonding to precious metals and their alloys. For strong and durable attachment to the aforementioned different substrate materials coexisting in the oral environment, it was anticipated that a combination of adhesive acid monomers and sulfur-containing monomers in multipurpose and adhesive primers would yield the desired result. Although a number of adhesive monomers have been included in the commercial dental adhesive forms, a recently published survey ${ }^{10,11}$ revealed poor judgment in the development of multiple-use initiators and adhesives containing acidic adhesive monomers and sulfur-containing monomers. ${ }^{10,11}$

\section{Adhesion: step by step}

In every adhesion process it is necessary to follow a series of steps, which ultimately seek to initially prepare the surfaces to adhere and finally achieve the appropriate adhesion between them.

\section{Preparation of the metal surface}

The most universal process for preparing the surface of a metal material is sandblasting with aluminum oxide. ${ }^{8}$ This process can be done in or out of the mouth. Sandblasting offers or produces a fresh, non-contaminating surface with a high surface energy and which can be easily wetted with suitable adhesive agents. ${ }^{9}$ Tom Blake studied the effect of the delay in the application of the adhesive after etching the metal surface and saw that this was of great importance in wetting. When it takes more than 24hours after the engraving, the HEMA does not moisten so well, presumably because an absorbent layer is created on the surface of the metal which reduces surface energy.

There are other techniques for preparing the surface of a metal, but in contemporary practice they are not used. These are: oxidation at high temperatures, immersion in an oxidizing agent, anodizing and treating the surface with a liquid alloy of gallium and tin. Another more recent form is the one known commercially as Rocatec, with which an ultra-fine mechanical retention is achieved through sandblasting with silica particles. ${ }^{10}$

The intraoral application is done with intraoral sand blasting machines, such as the Microetcher and later a specific silane is applied. Abrasion can be achieved on a metal surface with particles of 30 um in size..$^{10,11}$

\section{Metal bonding systems}

Once treated the surface of the metal the next step will be the application of an adhesive. The first generation of adhesives appeared in 1978, so first we will talk about mechanical retention systems, which were the only ones used until this time. ${ }^{11}$

Mechanical retention systems: ${ }^{11}$ Macromechanical retention can be achieved with holes or retentions, however this in many cases supposes aesthetic problems or marginal problems. This was solved using small spherical particles as a method of mechanical retention. Another way to achieve micro-retention is through electrochemical corrosion, which produces numerous small holes in the surfaces of metal alloys, especially nickel-chromium alloys. ${ }^{12}$

An example of macromechanical retention is what was done with the old bridges of Rochette. ${ }^{13}$ These were introduced in 1973 and were placed at the tooth contact points with the enamel engraved. Macromechanical retention was achieved thanks to some holes that were practiced, the retainers, which were later covered with composite and polymerized. ${ }^{13}$ The Rochette bridge had disadvantages, including the risk of fracture due to insufficient retention force and requiring considerable skill during placement due to the short polymerization time of the composite resin. To address these problems, the Maryland Bridge was developed in the late 1970s; It was easy to place and strong, since it does not use the retention holes in the retainers. However, it also had disadvantages: a complicated electrode etching procedure is required and can only be used with nickel-chromium alloys. ${ }^{14,15}$

First-generation adhesion systems: ${ }^{16-19}$ In 1978 the 4-META (4 methacryloyloxyethyl trimelliate anhydride) was developed. This was the first adhesive monomer that was effective for use with metal alloys. In 1982 he was released in Super - Bond C \& B, which contained 4 - META; And one year later, in 1983, Panavia EX containing $10-\mathrm{MDP}^{16}$ was developed. These adhesives are capable of adhering firmly to non - precious metal alloys and chromium - cobalt and nickel - chromium alloys. ${ }^{17}$ It was also desirable to develop adhesive primers which can be used with precious alloys because of the problems of allergies and cosmetic problems of non-precious alloys. ${ }^{18}$ Prior to the development of the noble alloying adhesive primers, the techniques were developed to produce an oxidized layer on the surface of the noble metal alloy, as this allowed the subsequent application of adhesive containing 4-META and MDP. ${ }^{18,19}$ These techniques include tin and the formation of an oxide layer by heating the surface of the precious metal alloy. ${ }^{19}$

Second generation adhesion systems: ${ }^{19-21}$ The first adhesive for precious alloys was VBATDT, which was first marketed by V - Primer in 1994. Another brand that offers it is Alloy Primer. The latter can be used in both precious and non-precious alloys. ${ }^{19}$ Many other adhesives for precious alloys appeared and were marketed after VBATDT.

They all contain sulfur, which seems to react with precious alloys. However, sulfur-containing monomers inhibit the polymerization of resins initiated by the BPO-amine redox systems. ${ }^{20}$ Therefore, Super-Bond C \& B is the preferred cement for use in combination with sulfur-containing primers, as it uses tri-n-butylborane (TBBO) as the initiator. ${ }^{20}$ As for adhesive primers for precious metal alloys: the effectiveness of each primer for each metal is different so it cannot be generalized about which would be better. Studies that have been done for $\mathrm{Ag}, \mathrm{Cu}, \mathrm{Pd}$ and $\mathrm{Au}$ have resulted in Zhang K et al. ${ }^{21}$

a) In V-Primer 8VBATDT) is effective for Ag.

b) Metaltite $8 \mathrm{MTU}-6$ ) for $\mathrm{Cu}$.

c) None is effective in your relationship with Pd.

Adhesion of metal to enamel or dentin: ${ }^{22,23}$ Having prepared the surface of the metal, it is necessary to prepare the surface of the enamel and/or dentine to adhere. The preparation of the enamel and dentin surface is achieved with etching orthophosphoric acid for 20seconds and subsequent drying. ${ }^{22}$ Adhesion can be achieved with a bipolar dentin adhesive such as HEMA. Between them an opaque luting is interposed, to avoid that the metal ${ }^{13}$ is visible.

Adhesion to ceramics: ${ }^{23}$ The ceramic is recorded with $9.6 \%$ hydrofluoric acid for two minutes. This acid is very corrosive. Then silane is applied as an adhesive agent for the ceramic and between both luting.

Adhesion to old resin: It is not common today, to get a solution to an old metal crown with resin on its front face. The only answer would be to remove it and make the new crown completely of resin, not ce- 
ramic, using the metallic base like coping. The engraving of the resin will be done with microether, the adhesive agent will be silane and between both surfaces a luting will be applied.

\section{Discussion}

Phosphonic acid monomers for adhesion to hard dental tissues, non-precious metal alloys and dental porcelains. Classically an adhesive monomer was defined as a polymerizable monomer having both hydrophilic and hydrophobic groups in its structure. However, most adhesive monomers that promote binding to precious metals do not have the hydrophilic group, but have a group containing sulfur atoms in place. Nowadays, an adhesive monomer is defined as a polymerizable monomer whose structure is constituted by three parts: a polymerizable functional group (PG), an adhesion promoting group (AG) and a connecting group, ${ }^{22}$ the latter being An organic group connecting the $\mathrm{PG}$ and $\mathrm{AG}$ groups. ${ }^{23}$

The bonding performance of an adhesive monomer depends on the ability of the adhesion promoting group to chemically interact with the tackifier. The adhesion promoting group may contain a sulfur atom for attachment to precious metal metals and alloys or a hydrophilic acid moiety for attachment to hard dental tissues and non-precious metal alloys. ${ }^{23,24}$ Starting from the premise that the molecular structure of an adhesive monomer consists of three parts, a study was carried out to investigate the effect of each group of binding properties. Among the synthesized experimental adhesive monomers, the aim was to determine the optimal molecular structure for adhesion. ${ }^{25} \mathrm{In}$ the $\mathrm{Ni}$ $\mathrm{Cr}$ alloy adhesion, three phosphate monomers (MEP: 26Mpa; MHP: 41Mpa; MDP: 44Mpa) showed higher adhesion strength than the other esters. Very developer, poor binding (0Mpa) was exhibited by the IEP having a non-polymerizable group, TEGMP having triethylene glycol as a hydrophilic spacer group, and MDS having sulfate group as an acid group. In conjunction with dentin, two 8MHP phosphate monoesters: 4.8MPa; MDP: 5.9) and an 8MDP-Bis phosphate diester: 4.7MPa) showed higher adhesion strength than the other esters. Therefore, the prerequisites of an optimal molecular structure for the effective bonding of the dentin and $\mathrm{Ni}-\mathrm{Cr}$ alloy should consist of a polymerizable group of methacryloyl, a hydrophobic spacer group and a phosphonic acid group. ${ }^{26}$

In 1974, Anber and Farley proposed vinylphosphonic acid (VPA) and vinylbenzylphosphonic acid (VBPA) for attachment to tooth structure. ${ }^{27}$ These phosphonic acid monomers exhibit minimal adhesion to hard dental tissues. ${ }^{27}$ Its low binding strengths are believed to be caused by its poor solubility in water and a non-flexible spacer alkylene group in its molecular structures, although it has an effective phosphonic acid group. During the last decade, however, interest in the use of phosphonic acid monomers has been Revived by the demonstration of its superior hydrolytic stability with improved bonding performance to hard dental tissues. ${ }^{27}$

The chemical interaction of phosphonic acid monomers with the dental substrate and the $\mathrm{Ni}-\mathrm{Cr}$ alloy is based on the carboxylic acid type interaction of hydroxyapatite - based adhesive monomers at the molecular level, it was speculated that the monomeric ionic phosphonic acid group Interact chemically as monomers bound with calcium cations of the hydroxyapatite, on the one hand, as well as with metallic oxides on the surface of $\mathrm{Ni}-\mathrm{Cr}$ alloy by another. ${ }^{28}$

Sulfur-containing monomers in the adhesion to precious metals and their alloys. Regarding the efficacy of sulfur-containing monomers in the attachment of dental resins to precious metals and their alloys, adhesive acid monomers exhibit poor adhesion to precious metals, such as gold, due to the chemical inertia of the precious metals. To achieve a strong adhesion to dental precious metal alloys, a number of surface modification methods have been developed: thermal treatment, electroplating with tin, silica coating, ion coating and the application of liquid Ga - Sn alloy. During the past 20years, sulfurcontaining monomers such as thiophenols, thiophosphoric acids, triazinedithiones, thiols, thiobarbituric acids, disulfides and thiouracil have been developed along with thiirane monomers. Primers containing these sulfur-containing monomers have two very desirable advantages: simple application procedure and favorable adhesion result. The latter advantages are derived from the ability of sulfurcontaining monomers to form chemical bonds with metal atoms on the surfaces of precious metals. ${ }^{29,30}$

\section{Dithio-octonoate monomers for adhesion to precious metals and their alloys:}

The results showed that all new dithio octonoate monomers exhibit high TBS to all precious metals and alloys used. No significant differences were observed in TBS between 6-MHDT, 10-MDDT, 12-MDDDT, 2-AEDT, MAEDT and EMEDT (24.7 to 29.3MPa) but showed significantly higher TBS 2-MEDT 17.2MPa) and BMMMDT (18.3MPa). Similarly, no significant differences in TBS were found between all dithio-octonoate monomers for silver (41.9 to $49.6 \mathrm{MPa}$ ) adhesion, platinum (36.8 to $47.6 \mathrm{MPa})$, palladium (36.7 To $47.5 \mathrm{MPa}$ ) and Au-alloy of Ag-Pd (35.0 to $48.8 \mathrm{MPa}$ ). The novel dithio-octonoate monomers exhibit high TBS to all metal adhesives tested after 2000 thermal cycles. These experimental results verified the excellent bondability of these new dithio - octonoate monomers to precious metals and alloys. ${ }^{30-34}$ Comparison between dithio-octonoate monomers and sulfur-containing monomers in combination with precious metals and their alloys.

In order to compare the binding performance of newly developed dithio-octonoate monomers (2-MEDT, 6-MHDT and 10-MDDT) against conventional sulfur-containing monomers, nine experimental monomers were used, based on non-cyclic disulfide (BMPDS) and bis (10 methacryloyloxydecyl) disulfide 8BMDDS9], a triazinedithione [6 (4-vinylbenzyl-n-propyl) amino] -1,3,5-bis (2-methacryloyloxethyl) disulfide (BMEDS), bis (5 methacryloyloxypentyl) disulfide -triazine-2,4-dithione (VBATDT9) and a thiophenol [N-84-mercaptophenyl) methacrylamide (MPMA) monomer. An acidic monomer, 4-META, was used as a control. Precious metals and their alloys used were $\mathrm{Au}, \mathrm{Pt}, \mathrm{Pd}, \mathrm{Ag}$ alloy and $\mathrm{Au}$ - Ag - Pd alloy. After the primer pretreatment and bonding by MMA - PMMA/BPO - TBS resins, to precious metals and alloys after 2000 thermal cycles (31). In conjunction with $\mathrm{Au}, \mathrm{Ag}$ alloy, and $\mathrm{Au}-\mathrm{Ag}$ Pd alloy, the three dithio-octonoate monomers (2-MEDT, 6-MHDT, 10-MDDT) exhibited significantly higher TBS than all conventional sulfur-containing monomers. In conjunction with gold alloys, 2 MEDT, 6 - MHDT, and 10 - MDDT exhibiting significantly higher TBS than BMEDS, BMPDS, VBATDT, MPMA and 4 - META (34) Thus, novel dithio-octonoate monomers exhibit excellent adhesion to precious metals and alloys when compared to conventional sulfurcontaining monomers. ${ }^{35}$

\section{Effects of the combination of acid monomers and sul- fur-containing monomers on adhesion to precious and non-precious metals and alloys ${ }^{36-39}$}

From the effects of the combination of VBATDT and MDP Suzuki et al added phosphoric acid monomer (MDP, $0.2 \%$ by weight) to an accelerator containing a triazinedithione monomer 8 VBATDT, 
$0.5 \%$ by weight). After pretreatment with MMA-PMMA molecules and TBBO resins, TBS to the precious metals and their alloys were measured after 2000 thermal cycles. The average bond strength at Au, $\mathrm{Ag}, \mathrm{Cu}$ and $\mathrm{Cr}$ was 25.2(3.6); 39.7(2.6); 31.1(10.00) and 34.2(9.7) respectively. ${ }^{36}$

In another study by Koizumi et al, a metal accelerator containing both VBATDT and MDP, was used with MMA-PMMA/TBBO resins for the binding of $\mathrm{Ag}-\mathrm{Pd}$ alloy and pure titanium (TiCP) $\mathrm{Cu}-\mathrm{Au} .{ }^{37}$ The treatment with the first alloy resulted from the binding resistance of Ag-Pd-Cu-Au alloy and Ti. VBATDT showed high TBS to $\mathrm{Cu}-$ metal, and the Ag-Pd-Cu-Au alloy contained $20 \%$ by mass of $\mathrm{Cu}$. Although the $\mathrm{Ag}-\mathrm{Pd}-\mathrm{Cu}-\mathrm{Au}$ alloy contained a high amount of precious metals $(\mathrm{Ag}+\mathrm{Pd}+\mathrm{Au}=78 \%$ by mass $)$, the primer alloy with resin/TBBO MMA - PMMA exhibits high bond strength to the alloy . These studies showed as a combination of monomers containing Sulfur and adhesive acid monomers capable of activating adhesive resins were able to adhere effectively to precious metals and nonprecious metals and their alloys. ${ }^{38}$

The interaction between VBATDT and precious metals was independent of the interaction between MDP and non-precious metals and that these interactions did not interfere with each other in their respective adhesion mechanisms. ${ }^{39}$

\section{Efficacy of metal primers in the adhesion of resin compounds to precious and non-precious metals and alloys $^{40-42}$}

Experimental metal primers containing a dithio-octanoate monomer (10 MDDT or 6 MHDT) and a phosphonic acid monomer (6-MHPA or 6 MHPP) were prepared to evaluate their effects on binding of a photopolymerizable composite resin prosthesis for metals Precious and not precious. ${ }^{40}$ After treating gold, gold alloys, silver alloys, Au - Ag - Pd alloys, and Ni - Cr alloys with the experimental primers, their SBSS with solidex were measured after 1 day storage followed by 5,000 thermal cycles. The results showed that when metallic adhesives were treated with the primer containing $5.0 \mathrm{wt} . \%$ Of 10 MDDT and 1.0 wt.\% Of 6 - MHPA, their SBSS ranged from 31.2(5.2) to $34.5(5.8) \mathrm{MPa}$, with a good Bonding durability after 5,000 thermal cycles. Thus, a combined application of a dithio-octonoate monomer and a phosphonic acid monomer provided effective solidex bonding of metals and alloys of precious and non-precious metals. ${ }^{41}$ The intensity of the exoelectron emission activity on the sandblast metal surfaces decreases with time in the air, ${ }^{22,23}$ resulting in a decrease in bond strength. However, when the sandblast metal surfaces were coated with a metal primer containing a dithio-octonoate monomer and a phosphonic acid monomer, there was no significant degradation of the bond strength, even after storage of 2 months in the air at $50^{\circ} \mathrm{C} .{ }^{42}$

\section{Adhesion to ceramics and metallic materials}

Silica-based ceramics, such as glass feldspar porcelains, are often used to plate metal structures or high strength ceramic cofias for ceramic restorations. ${ }^{43}$ Availability of improved dental ceramic materials, such as lithium disilicate, alumina-based ceramics (alumina), and zirconium oxide ceramics (zirconia) as core materials has led to widespread use of ceramic restorations during Last decade. ${ }^{23}$ Due to zirconia's favorable aesthetic characteristics, mechanical properties and biocompatibility, the use of ceramic systems based on zirconium oxide has become increasingly popular in dentistry, with polycrystalline yttrium tetragonal zirconia (Y-TZP) base materials for Prosthetic restorations of ceramic in fixed prosthesis. The clinical success of these restorations is based on a bond of solid and durable resin to restorative materials and Support structures of the tooth. ${ }^{44}$ However, the establishment of a strong and stable zirconia binding has been shown to be difficult. ${ }^{45}$

\section{Conclusion}

a) Phosphoric acid monomers provide sufficient demineralization of hard dental tissues, thereby providing better bonding to enamel and dentin.

b) Dithio-octonoate monomers exhibited better adhesion to metals and precious metal alloys than conventional sulfur-containing monomers.

c) For adhesion to metals and alloys of precious metals and non-precious metal alloys, the optimal combination of dithio-octonoate monomers and acidic adhesive monomers was determined to be $5.0 \mathrm{wt} \%$ of 10 MDDT and $1.0 \mathrm{wt} \%$ of 6 MHPA.

d) The combination of a silane coupling agent (3-MPTES), acidic adhesive monomers (6-MHPA, 6-MHPP and 4-MET) and dithio-octonoate monomers (6-MHDT and 10-MDDT) provided effective adhesion Dental porcelain, alumina, zirconia and gold alloys.

\section{Acknowledgements}

None.

\section{Conflict of interest}

Author declares that there is no conflict of interest.

\section{References}

1. Ikemura K, Tay FR, Endo T, et al. A review of chemical-approach and ultramorphological studies on the development of fluoride-releasing dental adhesives comprising new pre-reacted glass ionomer (PRG) fillers. Dent Mater J. 2008;27(3):315-339.

2. Thorat S, Diaspro A, Salern M. In vitro investigation of coupling-agent-free dental restorative composite based on nano-porous alumina fillers. J Dent. 2014;42(3):279-286.

3. Abousgelib MN, Kleverlaan CJ, Feilzer AJ. Selective infiltration-etching technique for a strong and durable bond of resin cements to zirconia-based materials. J Prosthet Dent. 2007;98(5):379-388.

4. Ikemura K, Tay FR, Nishiyama N, et al. Multi-purpose bonding performance of newly synthesized phosphonic acid monomers. Dent Mater J. 2007;26(1):105-115.

5. Viljanen E, Skrifvars M, Vallittu P. Dendritic copolymers and particulate filler composites for dental applications: degree of conversion and thermal properties. Dent Mater. 2007;23(11):1420-1427.

6. Conrad HJ, Seong WJ, Pesun IJ. Current ceramic materials and systems with clinical recommendations: a systematic review. J Prosthet Dent. 2007;98(5):389-404.

7. Koizumi H, Ishii T, Naito K, et al. Effects of triazine dithione and hydrophobic phosphate monomers on bonding to $\mathrm{Ag}-\mathrm{Pd}-\mathrm{Cu}-\mathrm{Au}$ alloy and titanium with a methacrylic resin-based luting agente. $J$ Adhes Dent. 2010;12(3):215-222.

8. Mohamed Hamouda I. Current perspectives of nanoparticles in medical and dental biomaterials. J Biomed Res. 2012;26:143-151. 
9. Park HJ. Biofilm-inactivating activity of silver nanoparticles: A comparison with silver ions. J Ind Eng Chem. 2013;19:614-619.

10. Cury JA, Tenuta LM. Enamel remineralization: controlling the caries disease or treating early caries lesions? Braz Oral Res. 2009;23(1):23-30.

11. Chaloupka K, Malam Y, Seifalian AM. Nanosilver as a new generation of nanoproduct in biomedical applications. Trends Biotechnol. 2010;28(11):580-588

12. Zhang K, Melo MA, Cheng L, et al. Effect of quaternary ammonium and silver nanoparticle-containing adhesives on dentin bond strength and dental plaque microcosm biofilms. Dent Mater. 2012;28(8):842-852.

13. Cheng L, Zhang K, Melo MA, et al. Anti-biofilm dentin primer with quaternary ammonium and silver nanoparticles. J Dent Res. 2012;91(6):598-604.

14. Zhang K, Cheng L, Wu EJ, et al. Effect of water-ageing on dentine bond strength and anti-biofilm activity of bonding agent containing new monomer dimethylaminododecyl methacrylate. J Dent. 2013;41(6):504-513.

15. Sun L, Chow LC, Frukhtbeyn SA, et al. Preparation and properties of nanoparticles of calcium phosphates with various $\mathrm{Ca} / \mathrm{P}$ ratios. J Res Natl Inst Stand Technol. 2010;115:243-255.

16. Moreau JL, Xu HK. Fluoride releasing restorative materials: Effects of $\mathrm{pH}$ on mechanical properties and ion release. Dent Mater. 2010;26(11):227-235.

17. You C, Han C, Wang X, et al. The progress of silver nanoparticles in the antibacterial mechanism, clinical application and cytotoxicity. Mol Biol Rep. 2012;39:9193-9201.

18. Li F, Weir MD, Chen J, et al. Comparison of quaternary ammonium-containing with nano-silver-containing adhesive in antibacterial properties and cytotoxicity. Dent Mater. 2013;29(4):450-461.

19. Chaloupka K, Malam Y, Seifalian AM. Nanosilver as a new generation of nanoproduct in biomedical applications. Trends Biotechnol. 2010;28(11):580-588.

20. Zhang K, Melo MA, Cheng L, et al. Effect of quaternary ammonium and silver nanoparticle-containing adhesives on dentin bond strength and dental plaque microcosm biofilms. Dent Mater. 2012;28(8):842-852.

21. Zhang K, Cheng L, Wu EJ, et al. Effect of water-ageing on dentine bond strength and anti-biofilm activity of bonding agent containing new monomer dimethylaminododecyl methacrylate. J Dent. 2013;41(6):504-513.

22. Moshaverinia A, Ansari S, Movasaghi Z, et al. Modification of conventional glass-ionomer cements with $\mathrm{N}$-vinylpyrrolidone containing polyacids, nano-hydroxy and fluoroapatite to improve mechanical properties. Dent Mater. 2008;24(10):1381-1390.

23. Weir MD, Chow LC, Xu HH. Remineralization of demineralized enamel via calcium phosphate nanocomposite. JDent Res. 2010;91(10):979-984.

24. Cheng L, Zhang K, Melo MA, et al. Anti-biofilm dentin primer with quaternary ammonium and silver nanoparticles. $J$ Dent Res. 2012;91(6):598-604.

25. Langhorst SE, O’Donnell JNR, Skrtic D. In vitro reminerali-zation effectiveness of polymeric ACP composites: quantitative micro-radiographic study. Dent Mater. 2009;25(7):884-891.

26. Schumacher GE, Antonucci JM, O'Donnell JNR, et al. The use of amorphous calcium phosphate composites as bioactive basing materials. Their effect on the strength of the composite/adhesive/dentin bond. $J$ Am Dent Assoc. 2007;138(11):1476-1484.

27. Yuko Takeoka, Miki Hayashi, Nami Sugiyama, et al. In situ preparation of poly (1-lactic acid-co-glycolic acid)/hydroxyapatite composites as artificial bone materials. Polymer Journal. 2015;47:164-170.
28. Wu SH, Li Y, Zhang YQ, et al. Porous titanium-6 aluminum-4 vanadium cage has better osseointegration and less micromotion than a poly-ether-ether-ketone cage in sheep vertebral fusion. Artificial organs. 2013;37(12):191-201.

29. Liu Y, Bao C, Wismeijer D, et al. The physicochemical/biological properties of porous tantalum and the potential surface modification techniques to improve its clinical application in dental implantology. Mater Sci Eng C Mater Biol Appl. 2015;49:323-329.

30. Fukuda A, Takemoto M, Saito T, et al. Osteoinduction of porous ti implants with a channel structure fabricated by selective laser melting. Acta Biomaterialia. 2011;7:2327-2336.

31. Van der Stok J, Van der Jagt OP, Amin Yavari S, et al. Selective laser melting-produced porous titanium scaffolds regenerate bone in critical size cortical bone defects. J Orthop Res. 2013;31(5):792-799.

32. Kokubo T, Takadama H. How useful is SBF in predicting in vivo bone bioactivity? Biomaterials. 2006;27(15):2907-2915.

33. Park JW, Kim YJ, Jang JH, et al. Effects of phosphoric acid treatment of titanium surfaces on surface properties, osteoblast response and removal of torque forces. Acta Biomater. 2010;6(4):1661-1670.

34. Minagar S, Wang J, Berndt CC, et al. Cell response of anodized nanotubes on titanium and titanium alloys. $J$ Biomed Mater Res A. 2013;101(9):2726-2739.

35. Whiteside P, Matykina E, Gough JE, et al. In vitro evaluation of cell proliferation and collagen synthesis on titanium following plasma electrolytic oxidation. J Biomed Mater Res A. 2010;94(1):38-46.

36. Diefenbeck M, M ckley T, Schrader C, et al. The effect of plasma chemical oxidation of titanium alloy on bone-implant contact in rats. Biomaterials. 2011;32(32):8041-8047.

37. Xie L, Liao X, Yin G, et al. Preparation, characterization, in vitro bioactivity, and osteoblast adhesion of multi-level porous titania layer on titanium by two-step anodization treatment. $J$ Biomed Mater Res A. 2011;98(2):312-320.

38. Zhang P, Zhang Z, Li W, et al. Effect of Ti-OH groups on microstructure and bioactivity of $\mathrm{TiO} 2$ coating prepared by micro-ark oxidation. Appl Surf Sci. 2013;268:381-386.

39. Shibata Y, Suzuki D, Omori S, et al. The characteristics of in vitro biological activity of titanium surfaces anodically oxidized in chloride solutions. Biomaterials. 2010;31(33):8546-8555.

40. Zhou R, Wei D, Yang H, et al. Osseointegration of bioactive microarc oxidized amorphous phase/ $\mathrm{TiO} 2$ nanocrystals composited coatings on titanium after implantation into rabbit tibia. J Mater Sci Mater Med. 2014;25(5):1307-1318.

41. Tsutsumi Y, Niinomi M, Nakai M, et al. Micro-arc oxidation treatment to improve the hard-tissue compatibility of Ti-29Nb-13Ta-4.6Zr alloy. Appl Surf Sci. 2012;262:34-38.

42. Yamaguchi S, Takadama T, Matsushita T, et al. Preparation of bioactive $\mathrm{Ti}-15 \mathrm{Zr}-4 \mathrm{Nb}-4 \mathrm{Ta}$ alloy from $\mathrm{HCl}$ and heat treatments after an $\mathrm{NaOH}$ treatment. J Biomed Mater Res Part A. 2011;97(2):135-144.

43. Yamaguchi S, Takadama H, Matsushita T, et al. Apatite-forming ability of $\mathrm{Ti}-15 \mathrm{Zr}-4 \mathrm{Nb}-4 \mathrm{Ta}$ alloy induced by calcium solution treatment. $J$ Mater Sci Mater Med. 2010;21:439-444.

44. Fukuda A, Takemoto M, Saito $T$, et al. Bone bonding bioactivity of $\mathrm{Ti}$ metal and $\mathrm{Ti}-\mathrm{Zr}-\mathrm{Nb}-\mathrm{Ta}$ alloys with $\mathrm{Ca}$ ions incorporated on their surfaces by simple chemical and heat treatment. Acta Biomater. 2011;7(3):1379-1386.

45. So K, Kaneuji A, Matsumoto T, et al. Is the bone-bonding ability of a cementless total hip prosthesis enhanced by alkaline and heat treatment? Clin Orthop Relat Res. 2013;471(12):3847-3855 